\title{
Intervenção do profissional de educação física nos Núcleos de Apoio à Saúde da Família em municípios do norte do Paraná
}

\author{
Professional intervention in Physical Education at the Health Family Support \\ Nucleus in municipalities in the North of Paraná
}

\section{Silvana Cardoso de Souza' \\ Mathias Roberto Loch ${ }^{1,2}$}

${ }^{1}$ Grupo de Estudos em Atividade Física, Saúde e Qualidade de Vida - GEAFSQ - Universidade Estadual de Londrina - PR

,2 Departamento de Educação Física Universidade Estadual de Londrina - PR

\section{Endereço para Correspondência}

\section{Mathias Roberto Loch}

Universidade Estadual de Londrina-

Departamento de Educação Física

Campus Universitário

Rodovia Celso Garcia Cid - PR 445, Km 380

Caixa Postal 6001 - CEP 86051-990

Londrina - Paraná - Brasil

e-mail:mathias@uel.br

$$
\begin{gathered}
\text { - Recebido: 21/5/2010 } \\
\text { - Re-submissão: } 17 / 07 / 2010 \\
\text { 06/08/2010 } \\
\text { - Aceito: 11/8/2010 }
\end{gathered}
$$

\begin{abstract}
Resumo
O objetivo deste estudo foi verificar as características de intervenção dos profissionais de Educação Física inseridos no Núcleo de Apoio à Saúde da Família (NASF). A amostra foi composta por sete profissionais que atuavam em municípios da região norte do estado do Paraná. Foi realizada uma entrevista semi-estruturada que foi gravada e transcrita posteriormente. Para analise das entrevistas foi utilizado elementos da analise de conteúdo através do sistema de categorias. Todos os profissionais formaram-se em Educação Física - Licenciatura Plena. Em relação aos cursos de graduação, os sujeitos referiram pouca aproximação deste com a área de saúde pública, de forma que, a maioria dos sujeitos recorre a novos estudos para desenvolver seu trabalho. Sobre os aspectos do trabalho em equipe os profissionais entendem que esta é uma grande possibilidade de troca de informações e que possibilita a realização de trabalhos coletivos. A intervenção na atenção básica é realizada na maioria dos casos através de grupos específicos de atividades físicas. Nestes, são desenvolvidos exercícios de alongamentos, fortalecimento muscular, caminhada, e em menor proporção exercícios de coordenação motora e atividades lúdicas. O número de pessoas atendidas nos grupos variou de 12 a 93 pessoas. A partir destes dados, percebe-se a necessidade de novos direcionamentos com o objetivo de aumentar a cobertura deste atendimento, uma vez que, deve a inserção do profissional de Educação Física na atenção primária ser um facilitador para o aumento das chances dos indivíduos serem fisicamente ativos.
\end{abstract}

Palavras chave: Atividade Física; Sistema Único de Saúde, Atenção primária.

\section{Abstract}

The aim of this study was to verify the characteristics of intervention of physical education professionals in Health Family Support Nucleus (NASF). The sample was formed by seven physical education professionals who worked in municipalities in the North of Paraná state. A semi-structured interviewed was held and also recorded and later transcribed.To analyse the interviews was used elements of the content analysis through the system of categories. All professionals graduated in Physical Education - Full Degree. In relation to graduation courses, subjects mentioned little of this approach to public health, so that, most of them tend to commit to new studies to develop their work. Concerning the aspects of team work the professionals understand that this is a great opportunity to exchange information and allows the realization of group work. The intervention in primary care is done in most cases by specific groups of physical activities. These are developed stretching exercises, muscle strengthening and walking, and in a reduced extent motor coordination exercises and ludic activities. The number of people benefited in the groups ranged from 12 to 93 people. From these data, we observe the need for new directions aiming at increasing the coverage of this service due to the professional insertion of Physical Education in primary care to be a facilitator to the increase of chances in individuals being physically active.

Keywords: Physical Activity, National Health Programs, Primary Health Care. 


\section{INTRODUÇÂO}

Diante da modificação das causas de mortalidade e de morbidade acontecidas no Brasil, o quadro de saúde pública tornou-se especialmente complexo, pois as doenças crônicas não transmissíveis passaram a ser hegemônicas, sem, entretanto o país ter resolvido seus problemas com as doenças infecto-contagiosas'.

Dado esse contexto, a criação do Sistema Único de Saúde (SUS) em 1990 representou um importante avanço em termos de saúde pública, em especial na atenção básica, uma vez que entre os objetivos da criação do SUS está a tentativa de substituição do modelo tradicional médico assistencial privado, que tinha o direcionamento centrado na cura da doença, para um modelo que visa, sobretudo, aspectos de promoção, prevenção e reabilitação ${ }^{2,3}$.

Tendo em vista tais direcionamentos, em 1994 o SUS teve suas ações fortalecidas através da implantação do programa Saúde da Família, atualmente referido como Estratégia Saúde da Família (ESF), com o objetivo de reorganizar a assistência à saúde e o processo de municipalização dos serviços. Vale lembrar, que programas com fins preventivos são considerados de baixo custo e quando bem estruturados, fortalecem o processo de prevenção das doenças e reduzem os gastos por internações ${ }^{4,5,6}$.

Ampliação importante da ESF aconteceu a partir do início de 2008, quando foi criado o Núcleo de Apoio à Saúde da Família (NASF), que incorpora profissões que até então não estavam inseridas nas Unidades Básicas de Saúde (UBS). Dada a complexidade do trabalho na atenção básica, devido à diversidade dos municípios e da população, espera-se que a união de diversos profissionais de diferentes áreas poderá proporcionar direcionamentos mais amplos, melhorando assim o sistema de saúde ${ }^{7,8,3}$.

Dentre as estratégias de promoção e prevenção de saúde a prática de atividades físicas tem sido referida como uma das ações importante nesse processo. Neste sentido, uma das áreas que passou a ter a possibilidade de inserção no NASF foi a Educação Física. Entretanto, na mesma medida que isto representa importante conquista para a área, não se pode perder de vista as possíveis dificuldades encontradas pelos profissionais recentemente inseridos, haja vista que de algum modo, estes são pioneiros e apresentam um papel histórico importante para a consolidação da área neste novo e importante contexto.

Dados estes apontamentos, este estudo teve como objetivo verificar as características de intervenção dos profissionais de Educação Física no NASF em municípios do Norte do Estado do Paraná.

\section{MÉTODOS}

Este estudo foi desenvolvido numa abordagem qualitativa do tipo exploratório-descritivo, buscando conhecer os aspectos particulares dos fatos que descrevem o trabalho do profissional de Educação Física inserido no NASF. Para isso, utilizou-se entrevista semi-estruturada, realizada entre os meses de agosto e setembro de 2009, através de uma série de perguntas abertas. Quando necessário foram acrescentadas outras questões para melhor esclarecimento.

O roteiro básico da entrevista incluiu: informações pessoais, percepções sobre o curso de graduação e informações sobre a intervenção no NASF. As entrevistas foram gravadas e após sua transcrição, apagadas. A transcrição das entrevistas totalizou 39 laudas.
A amostra foi selecionada de maneira intencional. O primeiro contato com os profissionais foi realizado por intermédio da secretaria municipal de saúde de cada município, e após a explicação sobre os objetivos do estudo, as entrevistas foram agendadas, conforme disponibilidade dos sujeitos.

O caráter de participação na pesquisa foi voluntário. Uma vez esclarecidos sobre os objetivos e procedimentos a serem adotados, os sujeitos tiveram a opção de não participar, não havendo nenhuma penalidade para aqueles que optassem pela não participação. Nenhuma recusa foi observada.

Todos os participantes assinaram o Termo de Consentimento Livre e Esclarecido concordando em participar com estudo. Os procedimentos metodológicos foram aprovados pelo Comitê de Ética e pesquisa com Seres Humanos da Universidade Estadual de Londrina, Parecer n ${ }^{\circ}$ 154/09.

A amostra foi composta por sete profissionais de Educação Física pertencente ao Núcleo de Apoio à Saúde da Família dos municípios de Apucarana, Cambé, Ibiporã e Rolândia, todos localizados na região norte do Paraná. O município de Londrina, maior município a região Norte do Paraná, não foi incluído neste estudo, pois no momento da coleta de dados, as atividades no NASF deste município estavam paralisadas.

Para analise das entrevistas foram utilizados elementos da analise de conteúdo através do sistema de categorias, que tem por objetivo proporcionar uma reprodução simplificada dos dados brutos ${ }^{9}$. As categorias foram divididas em três unidades temáticas: direcionamento do curso de graduação; intervenção profissional;e trabalho em equipe.

\section{RESULTADOS}

\section{Caracterização dos sujeitos}

Todos os entrevistados formaram-se em licenciatura plena, sendo que, cinco realizaram sua graduação em universidades públicas e dois na rede particular. Cinco concluíram curso de especialização. Em média os profissionais possuíam oito anos de formação, com variação entre dois e quatorze anos. $\mathrm{O}$ tempo de atuação no NASF variou de quatro meses a um ano.

\section{Entrevistas}

Para melhor compreensão dos resultados optou-se por dividir as analises em três blocos: no primeiro estão apresentadas as categorias que dizem respeito às características do curso de graduação; no segundo as categorias referentes ao trabalho em equipe; e no terceiro as categorias quanto à forma de organização e tipo de atividades desenvolvidas no NASF. Para melhor ilustração das explicações serão apresentados trechos das falas dos sujeitos. Vale lembrar que a transição dos trechos ocorreu de forma integra de acordo com as falas.

O quadro 1 apresenta as categorias relacionadas ao direcionamento dos cursos de graduação. Três dos sujeitos mencionaram que seu curso de graduação tinha foco centrado no esporte; dois afirmaram falta de aproximação com a área de saúde pública e dois relataram que durante sua graduação tiveram disciplinas que deram base para intervenção em saúde, (apesar de não serem disciplinas específicas sobre a organização da saúde pública, mas sim disciplinas como prescrição de exercícios, entre outras).

Através do relato dos entrevistados pode-se perceber que embora alguns dos sujeitos não identificassem a forte influência do esporte durante seu curso de graduação, mencionaram pouca aproximação deste com a área de saúde pública, tendo que recorrer a novos estudos para desenvolver seu trabalho neste ambiente. Tais afirmativas são reforçadas 


\begin{tabular}{|c|c|c|c|c|c|c|c|}
\hline Categorias & S 1 & S 2 & S 3 & S 4 & S 5 & S 6 & S 7 \\
\hline Esporte & $\mathrm{X}$ & & $X$ & & & $\mathrm{X}$ & \\
\hline $\begin{array}{c}\text { Ausência de Saúde } \\
\text { Pública }\end{array}$ & & & & $\mathrm{X}$ & & & $X$ \\
\hline Saúde & & $\mathrm{X}$ & & & $\mathrm{X}$ & & \\
\hline
\end{tabular}

\section{Quadro 1}

\section{Caracterização do trabalho em equipe, segundo percepção dos sujeitos entevistados}

pelas falas dos sujeitos:

S 1: [...] meu curso de graduação não me deu muita base para trabalhos externos a não ser treinamento esportivo [...].

S 3: [...] nós éramos um clubão ali, foi talvez melhor do que os outros que eu ouvi dizer, mais eu acredito que muito pouco, apesar da pouco experiência que tenho nessa parte eu acredito que se foi, foi pouca.

S 4: [...] Não tive saúde pública na faculdade eu tive que estudar mais[...].

S 6: [...] Olha quando eu entrei na graduação foi bem relacionado com a relação ao esporte, eu praticava ciclismo naquela época então eu tinha uma visão bem da educação física junto ao esporte... lá se fala em educação física relacionada a saúde, não tem educação física relacionada a saúde pública que é outra situação[...].

Outro argumento relatado pelos entrevistados refere-se à importância de buscar novos conhecimentos para atuação nas UBS como os apresentados nas falas a seguir:

S 1: [...] Eu acredito que os profissionais deviam se ater a estudar um pouco mais a saúde pública e a questão de treinamentos novos para esse pessoal por que é uma população muito carente é uma população carente de atenção é uma população carente de toda atividade que se possa ser realizado com eles... acho que as escolas as instituições as escolas de educação física deveriam propiciar um conhecimento mais na área de saúde pública mais enquanto isso não se tem os profissionais deviam procurar isso com novas experiência [...].

S 3: [...] eu acho que nós profissionais da atividade física, eu não sei como que tá hoje por que faz muitos anos que eu sai... acho que a gente tem que tá estudando bastante. Acho que temos que valorizar nossa profissão. Temos que buscar mais, na minha época era assim a gente tinha pouca teoria e eu também não me aprofundei mais nesse sentido, mas acho que a gente tem que buscar mais conhecimento, acho que mais conhecimento vai valorizar nossa profissão. [...].

O quadro 2 refere-se a visão dos profissionais sobre o trabalho interdisciplinar, sendo considerado por estes uma grande possibilidade de troca de conhecimentos. Além disso, seis dos sujeitos mencionaram que esta experiência possibilita a realização de trabalho coletivo.

Para exemplificar tais afirmações serão apresentados trechos das respostas de alguns profissionais:

S1: [...] achei interessante por que a gente conversa entre os profissionais e trocamos informações [...].

S 4: [...] nós vamos trabalhar promoção e prevenção, então

\begin{tabular}{|c|c|c|c|c|c|c|c|}
\hline Categorias & S 1 & S 2 & S 3 & S 4 & S 5 & S 6 & S 7 \\
\hline $\begin{array}{c}\text { Troca de } \\
\text { Conhecimento }\end{array}$ & $\mathrm{X}$ & $\mathrm{X}$ & $\mathrm{X}$ & $\mathrm{X}$ & $\mathrm{X}$ & $\mathrm{X}$ & $\mathrm{X}$ \\
\hline $\begin{array}{c}\text { Trabalho } \\
\text { Coletivo }\end{array}$ & $\mathrm{X}$ & $\mathrm{X}$ & $\mathrm{X}$ & $\mathrm{X}$ & $\mathrm{X}$ & $\mathrm{X}$ \\
\hline
\end{tabular}


pra comunidade é muito importante ter todos esse profissionais envolvidos e a gente discute mesmo entre nós... a gente acaba trocando as informações com outros profissionais, por que você não vai ver só o lado da atividade física, do exercício você troca informações com o farmacêutico, os medicamentos que aquele hipertenso toma, diabéticos... então a gente consegue ter toda uma rede de informações [...].

\section{S 5: [...] traz um feedback legal, outras idéias outros conhe-} cimentos [...].

S 6: [...] é como se... tudo aquilo que você aprendeu e sabe da educação física você vai ter que repartir isso com a equipe por que você vai ter uma área a ser cuidada.... e isso é muito gratificante é muito prazeroso... você cresce profissionalmente, você cresce como pessoa, você cresce como homem [...].

\section{S 7: [...] é um trabalho bem legal eu nunca tinha vivido isso} na minha vida e foi assim uma coisa louca, uma novidade tremenda na minha vida nunca imaginei que eu fosse tá em meio assim eu, e outros profissionais por que assim igual eu falei foi sempre "eu", eu dentro duma academia por mais que eu tivesse dentro de uma escola era eu dentro de uma quadra... hoje eu me vejo junto com outras pessoas com outros pensamentos, mas todo mundo no final falando a mesma língua. Entendeu? É isso que eu achei bem legal bem interessante.

Essas características são observadas e comentadas, inclusive por um dos profissionais que não tem o suporte do trabalho em equipe. Neste caso específico, cada profissional que compunha o NASF trabalhava de maneira isolada.

S 3: [...] quando você fala assim equipe do NASF tem que tá uma equipe ... não ter todo mundo junto no mesmo lugar mais ter uma troca pelo menos uma troca de um dia na semana[...].

Além disso, os profissionais mencionaram que no início encontraram dificuldades devido à resistência por parte de alguns profissionais da ESF. Entretanto, segundo os relatos, parte deste problema foi resolvido a partir da capacitação realizada com os profissionais das UBS, em especial, as agentes comunitárias de saúde.

A seguir serão apresentados os resultados encontrados em relação à forma de intervenção profissional (quadro 3). Vale destacar que, em média, cada profissional atuava em cinco unidades fixas e seu trabalho era realizado por escala. Dos sujeitos entrevistados seis realizam seu trabalho através de grupos nas unidades e apenas um atuava exclusivamente dando suporte as necessidades de cada UBS, em especial, a equipe da ESF.

Além das atividades físicas a maioria dos profissionais referiu realizar mini-palestras nos grupos sobre temas relacionados à importância da prática de atividade física. A participação nos grupos é voluntária e a divulgação é feita através de cartazes nos postos de saúde e pelos profissionais da ESF, sendo essa realizada dentro da própria unidade. Dois profissionais afirmaram realizar visitas domiciliares convidando a população a participar dos grupos, conforme falas a seguir:

S 1: [...] na educação física nos montamos grupos, convidamos através das Agentes comunitárias, elas fazem convite a população pra estarem junto conosco nas unidades pra esse treinamento pra esses grupos [...].

S 4: [...] as pessoas são orientadas nas unidades. A gente usa estratégia de divulgar por cartazes. Eu mesmo faço as visitas domiciliares pra falar da importância da atividade física, do exercício e estar convidando essas pessoas pra tá participando das atividades [...].

Dentre as atividades físicas prescritas pelos profissionais estão: atividades aeróbias (principalmente caminhadas) e exercícios resistidos (realizados com materiais alternativos como, por exemplo: pesos feitos de garrafa pet com areia ou pedra, cabo de vassoura dentre outros). Em menor proporção, os profissionais também referiram prescrever exercícios de alongamento, coordenação motora e atividades lúdicas. A maioria da população participante em todos os grupos são mulheres e idosos, normalmente com alguma patologia.

Em relação ao controle da intensidade das atividades, todos os profissionais afirmaram levar em consideração a percepção de cada indivíduo. As atividades são desenvolvidas de duas a três vezes por semana com aproximadamente uma hora de duração, sendo estas divididas em: aquecimento, treinamento especifico e relaxamento/alongamento.

O número de pessoas atendidas nos grupo variou de 12 a 93 pessoas e a principal dificuldade mencionada pelos profissionais para o desenvolvimento das atividades corresponde à falta de espaços, conforme falas a seguir:

S 4: [...] como a gente procura usar espaços públicos, uma certa dificuldade as vezes numa quadra que não tem uma estrutura boa ou ginásio. Nós usamos, mas nós fazemos

\begin{tabular}{|c|c|c|c|c|c|c|c|}
\hline Categoria & S 1 & S 2 & S 3 & S 4 & S 5 & S 6 & S 7 \\
\hline $\begin{array}{c}\text { Montando } \\
\text { Grupos }\end{array}$ & $\mathrm{X}$ & $\mathrm{X}$ & $\mathrm{X}$ & $\mathrm{X}$ & $\mathrm{X}$ & & $\mathrm{X}$ \\
\hline $\begin{array}{c}\text { Dando } \\
\text { Suporte às } \\
\text { UBS* }\end{array}$ & & & & & $\mathrm{X}$ & \\
\hline
\end{tabular}

* UBS= Unidade básica de saúde. 
caminhadas nas ruas, aí tem toda a estrutura de calçada... mas não é por isso que a gente vai deixar de fazer, mais a gente fica limitado.

S 7: [...] as vezes eu preciso de um espaço, geralmente é uma quadra e no calor. Seria ideal uma quadra coberta e nem é sempre que tem disponível. No município existem vários outros setores trabalhando no caso área do esporte, que é a secretaria do esporte e eles oferecem horários pra quadra. Então quando foi implantado o NASF a maioria das quadras já estavam com os horários todos preenchidos ... então esse é um problema: eu ter que adaptar lugar, improvisar lugar..., algumas vezes é na rua próximo as unidades porque eu também não posso me deslocar.

Em relação às estratégias para mobilização da comunidade, a maior parte dos sujeitos não referiu nenhuma ação específica, como por exemplo, a realização de grandes eventos, que busquem chamar a atenção para a importância da prática de atividade física, ou mesmo para divulgar as ações do NASF nas respectivas comunidades.

\section{DISCUSSÃO}

Este trabalho buscou verificar as características da intervenção de profissionais de Educação Física em um novo contexto de atuação (Núcleo de Apoio à Saúde da Família). Vale destacar que a escolha pela análise de conteúdo, como meio para análise das informações, se deveu por esta possibilitar um maior aprofundamento da realidade, e uma aproximação maior dos sujeitos investigados, que podem ser considerados importantes "pioneiros" da área.

A análise das entrevistas reforça a idéia que a Educação Física tem uma grande trajetória a ser construída na atenção básica à saúde, tanto no que diz respeito à inclusão profissional, como também à melhor formação deste. Vale mencionar que a formação demasiadamente "esportivizada", referida por alguns dos sujeitos, remete à história da Educação Física no Brasil $^{10,11}$.

Mesmo com algumas mudanças recentes ocorridas nos cursos de graduação, a presença de currículos generalistas superficiais, na maioria das vezes não oferecia uma base sólida para a intervenção profissional, pois ao mesmo tempo em que se objetivava formar o professor para atuar no contexto escolar, também se buscava a formação de profissionais para intervir fora deste ambiente ${ }^{12}$.

Nesse sentido, pode-se considerar a divisão dos cursos em licenciatura e bacharelado como uma alternativa de meIhor caracterizar o campo de intervenção para cada habilitação. Entretanto, quanto à intervenção na saúde pública trata-se de uma formação que ainda deve ser consolidada. Anjos et al, (2009) ${ }^{13}$ analisaram currículos dos cursos de bacharelado em Educação Física em universidades do estado de São Paulo, e constaram que ainda é deficiente a formação para atuação em saúde pública. Além disso, as disciplinas que mais se aproximam para tal intervenção restringiam-se ao caráter curativo e de prescrição, havendo pouca aproximação com a formação para o trabalho em uma abordagem coletiva. Essa realidade, também observada no presente estudo, merece especial atenção tendo em vista que a preocupação de formar profissionais aptos para intervir nesse setor é uma realidade e apresentada inclusive nas diretrizes do Ministério da Educação ${ }^{14}$.

Vale lembrar também que o trabalho no contexto do NASF é desenvolvido em uma equipe, o que se justifica inclusive pela complexidade do trabalho na atenção básica em saúde. Peduzzi, (2001) ${ }^{15}$ refere que a elaboração de projetos comuns se configura uma importante estratégia a ser adotada pelas equipes com o intuito de abranger às necessidades e a realidade de cada área a ser atendida.

Quanto às atividades desenvolvidas pelos profissionais nas UBS, seis dos sujeitos realizam seu trabalho no NASF através de atividades físicas desenvolvidas em grupos, com o auxilio de materiais alternativos que são uma opção de baixo custo. Observação parecida já havia sido apresentada por Kokubun, et al. (2007) ${ }^{16}$, que investigou a atuação na Estratégia Saúde da Família, mas não na perspectiva do NASF.

Entretanto, quando comparado o número de atendimentos nos grupos com a quantidade de pessoas na comunidade, observa-se que o número relativo é pequeno. Tal resultado pode ser em parte atrelado ao fato de que nem todos os profissionais realizam visitas domiciliares, convidando a população a participar. Além disso, há certa deficiência em relação a eventos que visem mobilizar o município da importância da prática de atividade física de forma que, as informações oferecidas pelos profissionais do NASF, acabam ficando restritas aos indivíduos que participam dos grupos. É claro que não se pode atribuir a falta de participação unicamente a estes fatos, uma vez que são múltiplos os fatores que influenciam na adesão o não a programas de atividade física ${ }^{17,18,19}$.

Vale lembrar também que uma das dificuldades na realização das atividades citadas pelos profissionais, consiste na falta de espaços adequados para a execução das mesmas, o que reforça a idéia de que devem ser múltiplas as estratégias para diminuição da prevalência de sedentarismo, inclusive com mudanças no ambiente.

Além disso, quando uma nova proposta é feita à comunidade, é normal que não haja plena adesão logo em seu início. Jenkins (2007) ${ }^{20}$ menciona cinco grupos comunitários teóricos sucessivos em termos de seqüência na adoção da "novidade", sendo eles: Inovadores (aqueles que primeiro irão aderir), aprovadores iniciais, maioria inicial, maioria tardia e os resistentes remanescentes (que mais dificilmente aderem).

Outro achado importante no estudo refere-se que grande parte dos indivíduos que freqüentam os grupos são muIheres. Achados semelhantes foram observados por Hallal el al, $(2010)^{21}$, na cidade de Recife, onde a maior parte dos usuários do programa Academia da Cidade, programa este com especificidades diferentes do NASF, era mulheres.

Vale destacar que a criação do NASF é uma tentativa de possibilitar um direcionamento mais amplo das ações de saúde pública. Para tanto, faz-se necessário que o trabalho não tenha apenas um direcionamento "clinico", pois como documentado na própria resolução: "as ações de Atividade Física/ Práticas Corporais devem buscar a inclusão de toda a comunidade adstrita, não devendo restringir seu acesso apenas às populações já adoecidas ou mais vulneráveis"8.

\section{CONCLUSÃO}

O trabalho em saúde pública, especialmente em um contexto complexo como o brasileiro, consiste em uma tarefa bastante difícil. A inclusão de outras profissionais ao quadro tradicional pode ser de grande importância para a construção de um enfoque mais ampliado de saúde.

No caso da Educação Física, alguns aspectos devem ser considerados, pois podem representar dificuldade para a inserção e a consolidação da área neste novo campo de intervenção. Um dos fatos a ser destacado é o direcionamento dos cursos de graduação generalista para a área esportiva, em 
detrimento a uma pequena aproximação da área de saúde pública. Espera-se que com a nova formação (dividida entre licenciatura e bacharelado), os conteúdos relacionados à saúde pública sejam mais aprofundados durante a graduação.

Além disso, a maioria dos profissionais investigados referiu desenvolver sua intervenção através de grupos específicos, onde apenas uma pequena parcela da população participa. Neste sentido, faz-se urgente que novos direcionamentos sejam tomados, com o com o intuito de aumentar a cobertura deste atendimento. Deve a inserção do profissional de Educação Física ser um facilitador no sentido de aumentar a chance dos indivíduos serem fisicamente através da criação de diferentes estratégias que enfoquem o conhecimento, as atitudes e os comportamentos da população atendida.

Um chamado interessante seria a realização de um entrosamento entre os municípios, inclusive defendido pelos preceitos do NASF, para uma possível transferência de experiências com o objetivo de melhorar a intervenção dos profissionais, evitando assim a descaracterização do projeto.

Estudos futuros poderiam acompanhar a dinâmica de trabalho dos profissionais, visando inclusive registrar o impacto da inserção deste sobre os indicadores de saúde da população.

\section{Agradecimentos}

As Profs. Rubiane Giovane Fonseca e Prof. Dra. Jeane Barcelos Soriano, pelo auxílio na análise dos dados e pelas contribuições metodológicas.

\section{Contribuições dos autores}

Silvana Cardoso de Souza coletou os dados, transcreveu as entrevistas, analisou os dados e escreveu o artigo. Mathias Roberto Loch orientou todas as etapas da pesquisa e realizou a revisão final do artigo.

\section{REFERÊNCIAS}

1. Schramm JMA, Oliveira AF, Leite IC, et al. Transição epidemiológica e o estudo de carga de doença no Brasil. Cienc Saúde Coletiva. 2004; 9(4):897-908.

2. Florindo AA. Núcleos de apoio à saúde da família e a promoção das atividades físicas no Brasil: de onde viemos onde estamos e para onde vamos. Revista Brasileira de Atividade Física e Saúde, 2009; 14:2.72-73.

3. Hortale VA, Conill EM, Pedroza M. Desafios na construção de um modelo para análise comparada da organização de serviços de saúde. Caderno de Saúde Pública 1999;15:79-88.

4. Brasil, Ministério da Saúde. Saúde da Família: uma estratégia para a reorientação do modelo assistencial. Brasília, Governo Federal: Ministério da Saúde 1994.

5. Oliveira AKP, Borges DF. Programa de Saúde da Família: uma avaliação de efetividade com base na percepção de usuários. Revista de Administração Pública 2008;42:2:369-389.

6. Santana ML, Carmagnani MI. Programa saúde da família no Brasil: um enfoque sobre seus pressupostos básicos, operacionalização e vantagens. Saúde e Sociedade 2001;10:1:33-53.

7. Almeida $\mathrm{MCP}$, Mishima SM. O desafio do trabalho em equipe na atenção à saúde da família: construindo novas autonomias no trabalho. Interface 2001:59:150-153.

8. Brasil, Ministério da Saúde. Núcleo de Apoio da Saúde da Família. Portaria Ministerial de Saúde n¹54, de 24 de janeiro de 2008. Acessado em 21 de abril de 2009. Disponível em http://www.saude.sc.gov.br/PSF/PORTARIAS/ PORTARIA\% 20GM\%20N154.pdf.

9. Bardin L. Análise de Conteúdo. Lisboa: Edições 70, 1977.

10. Almeida MB, Gutierrez GL. O lazer no Brasil: do nacional-desenvolvimentismo à globalização. Conexões 2005;3:1:36-57.

11. Ghilardi R. Formação profissional em educação física: a relação teoria e prática. Motriz 1998; 4:1:1-11.

12. Fávaro PE, Nascimento GY, Soriano JB. O conteúdo da intervenção profissional em Educação Física: o ponto de vista de docentes de um curso de formação profissional. Revista Movimento 2006; 12:2:199-221.

13. Anjos TC, Duarte ACGO. A educação física e a estratégia saúde da família: formação e atuação profissional. Revista de Saúde Coletiva 2009; 191127-1144.

14. Ministério da Educação: disponível em www.mec.gov.br/ acessado em 30/05/2010.

15. Peduzzi M. Equipe multiprofissional de saúde: conceito e tipologia. Revista de Saúde Pública 2001;1:35:63-72.

16. Kokubun $E$, Luciano $E$, Sibuya $C Y$, et al. Programa de atividade física em unidades básicas de saúde: relato de experiência no município de Rio Claro - SP. Revista Brasileira de Atividade Física e Saúde 2007;12:1:45-53.

17. Gobbi S, Cataritá LP, Hirayama MS, et al. Comportamento e Barreiras: Atividade Física em Idosos Institucionalizados. Psicologia: Teoria e Pesquisa 2008; 24:4: 451-458.

18. Nahas MV. Atividade Física, saúde e qualidade de vida: conceitos e sugestões para um estilo de vida ativo. Londrina: Midiograf, 2006.

19. Santos SC, Knijinik JD. Motivos de adesão à prática de atividade física na vida adulta intermediária. Revista Mackenzie de Educação Física e Esporte 2006;5:23-34.

20. Jenkins $C D$. Construindo uma saúde melhor: um guia para mudança de comportamento. Porto Alegre: Artmed,2007.

21. Hallal PC, Tenório MCM, Tassiano RM, et al. Avaliação do programa de promoção da atividade física Academia da Cidade de Recife, Pernambuco, Brasil: percepções de usuários e não-usuários. Cad. Saúde Pública 2010;26:1: 70-78. 